\title{
The Glasgow alcoholic hepatitis score identifies patients who may benefit from corticosteroids
}

\author{
E H Forrest, A J Morris, S Stewart, M Phillips, Y H Oo, N C Fisher, G Haydon, J O'Grady, C P Day
}

Gut 2007;56:1743-1746. doi: 10.1136/gut.2006.099226

See end of article for authors' affiliations

Correspondence to: Dr Ewan H Forrest, Department of

Gastroenterology, Glasgow Royal Infirmary, Castle Street, Glasgow G4 OSF; ewan.forrest@northglasgow. scot.nhs.uk

Revised 21 June 2007 Accepted 3 July 2007 Published Online First 25 July 2007

\begin{abstract}
Introduction: There is no consensus on the pharmacological treatment of alcoholic hepatitis. The Glasgow alcoholic hepatitis score (GAHS) has been shown to be more accurate than the modified Maddrey's discriminant function (mDF) in the prediction of outcome from alcoholic hepatitis. This study aimed to determine whether the GAHS was able to identify those patients who would benefit from corticosteroids.

Methods: 225 patients with an mDF greater than or equal to 32 from five hospital centres in the United Kingdom were reviewed. Patient survival relative to the GAHS and the use of corticosteroids was recorded. Results: 144 patients with an mDF greater than or equal to $32(64 \%)$ also had a GAHS greater than or equal to 9. There was no difference in survival between untreated or corticosteroid-treated patients for those with a GAHS less than 9. For patients with a GAHS greater than or equal to 9 the 28-day survival for untreated and corticosteroid-treated patients was $52 \%$ and $78 \%(p=0.002)$, and 84 -day survival was $38 \%$ and $59 \%$ $(p=0.02)$, respectively.

Conclusions: Among patients with an mDF greater than or equal to 32, there was no appreciable benefit from treatment with corticosteroids in patients with a GAHS less than 9. Patients with a GAHS greater than or equal to 9 have an extremely poor prognosis if they are not treated with corticosteroids, or if such treatment is contraindicated.
\end{abstract}

A lcoholic hepatitis is an increasingly common reason for hospital admission, and patients with severe alcoholic hepatitis are recognised to have a high short-term mortality. The severity of alcoholic hepatitis is often assessed using the modified Maddrey's discriminant factor (mDF). An $\mathrm{mDF}$ greater than or equal to 32 is associated with a $68 \% 28$-day survival in placebo-treated patients whereas those with a score less than 32 have a survival of $93 \%$.

We have recently described the Glasgow alcoholic hepatitis score (GAHS) for the assessment of patients presenting with a clinical diagnosis of alcoholic hepatitis. This score was derived from a population of 241 patients from Glasgow and validated in a separate cohort of 195 patients from throughout the United Kingdom $^{2}$ (table 1). None of these patients had received corticosteroids, pentoxifylline or anti-tumour necrosis factor treatment. Our analysis indicated that the GAHS was more accurate in predicting outcome at 28 and 84 days after admission, and was more specific for death, when compared with the $\mathrm{mDF}$.

Controversy continues to overshadow the pharmacological treatment of alcoholic hepatitis. Despite numerous controlled trials and meta-analyses of corticosteroid treatment in alcoholic hepatitis there is little international consensus upon its use. ${ }^{3-5}$

Table 1 The Glasgow alcoholic hepatitis score

\begin{tabular}{llll}
\hline Score given & $\mathbf{1}$ & $\mathbf{2}$ & $\mathbf{3}$ \\
\hline Age $\left(10^{9} / \mathrm{l}\right)$ & $<50$ & $\geqslant 50$ & - \\
WCC & $<15$ & $\geqslant 15$ & - \\
Urea $(\mathrm{mmol} / \mathrm{l})$ & $<5$ & $\geqslant 5$ & - \\
PT ratio or INR & $<1.5$ & $1.5-2.0$ & $>2.0$ \\
Bilirubin $(\mu \mathrm{mol} / \mathrm{l})$ & $<125$ & $125-250$ & $>250$ \\
\hline
\end{tabular}

INR, International normalised ratio; PT, prothrombin time; WCC, white cell count.

Each variable is given a score and then a combined score of between 5 and 12 is obtained. A score greater than or equal to 9 is associated with a poor prognosis.
The American College of Gastroenterology has recommended that the $\mathrm{mDF}$ be used to assess the severity of alcoholic hepatitis and a threshold of 32 be used to consider corticosteroid therapy. ${ }^{6}$ A re-analysis of three large randomised controlled studies investigating corticosteroid use in alcoholic hepatitis indicated a likely improvement in 28-day survival from $65.1 \%$ to $84.6 \%{ }^{5}$ among patients with an $\mathrm{mDF}$ greater than or equal to 32.

Having established the GAHS as an accurate predictor of outcome in alcoholic hepatitis we aimed not to assess the effectiveness of corticosteroids, but rather to assess the role of the GAHS in identifying those patients who might benefit from pharmacological intervention with corticosteroids.

\section{METHODS}

As part of the process to validate the GAHS we identified patients presenting to hospitals in five hospital centres within the United Kingdom: Glasgow, Newcastle, London (King's College), Birmingham, and Dudley. Patients from Newcastle and London were part of randomised controlled trials. ${ }^{78}$ All patients had a serum bilirubin level greater than or equal to $80 \mu \mathrm{mol} / \mathrm{l}$ and a history of excessive alcohol ingestion (greater than or equal to $50 \mathrm{~g} /$ day) until at least three weeks before admission. As in the initial dataset, patients with viral hepatitis, autoimmune liver disease, biliary obstruction or hepatocellular carcinoma were excluded.

The GAHS was calculated for each patient on the day of admission. In 35 patients all the variables were not available and the GAHS was classified as greater than or equal to or less than 9 for 25 patients for whom it was arithmetically possible ( 12 in the corticosteroid-treated group). The 10 patients whose GAHS score could not be categorised were excluded from further analysis. Only patients who would normally be

Abbreviations: GAHS, Glasgow alcoholic hepatitis score; INR, international normalised ratio; mDF, modified Maddrey's discriminant factor; MELD, model of end-stage liver disease 
Table 2 Selection of patients for validation of the Glasgow alcoholic hepatitis score

\begin{tabular}{|c|c|c|c|c|}
\hline Hospital site & $\begin{array}{l}\text { No. of patients: total } \\
\text { (NT; CS) }\end{array}$ & Dates of recruitment & Inclusion/exclusion criteria & Indications for corticosteroids \\
\hline Glasgow & $38(8 ; 30)$ & $2002-3$ & GI bleeders excluded & $\begin{array}{l}\mathrm{CS} \text { used if sepsis excluded or adequately } \\
\text { treated; } m D F \geqslant 32\end{array}$ \\
\hline Birmingham & $25(19 ; 6)$ & $2002-3$ & GI bleeders excluded & $\begin{array}{l}\text { CS used if sepsis excluded or adequately } \\
\text { treated; } \mathrm{mDF} \geqslant 32\end{array}$ \\
\hline Newcastle & $65(29 ; 36)$ & $2000-3$ & $\begin{array}{l}\text { Part of an } \mathrm{RCT} \text { comparing antioxidants with } \\
\text { placebo; } \mathrm{mDF} \geqslant 32\end{array}$ & CS used if no active infection or GI bleeding \\
\hline London & $82(38 ; 44)$ & 1997-2001 & $\begin{array}{l}\text { RCT comparing antioxidants with CS; mDF } \\
\geqslant 32 \text {; serum bilirubin }>100 \mu \mathrm{mol} / \mathrm{l} \text {, serum } \\
\text { creatinine }<500 \mu \mathrm{mol} / \mathrm{l} \text {. No active sepsis or } \\
\text { Gl bleeding for } 48 \text { hours }\end{array}$ & Randomised as part of RCT \\
\hline Dudley & $15(14 ; 1)$ & $2001-3$ & Patients with recent Gl bleeding included & CS not used routinely \\
\hline
\end{tabular}

CS, Corticosteroids ( $40 \mathrm{mg}$ prednisolone for 4 weeks except for London where $30 \mathrm{mg}$ was given for 4 weeks); $\mathrm{Gl}$, gastrointestinal; mDF, modified Maddrey's discriminant factor; NT, not treated; RCT, randomised controlled trial;

All patients had a serum bilirubin $\geqslant 80 \mu \mathrm{mol} / \mathrm{l}$, a history of recent alcohol excess and aspartate aminotransferase level $<500 \mathrm{IU} / \mathrm{l}$.

considered for treatment with corticosteroids were included, thus all patients described had an mDF greater than or equal to 32 on admission. Outcome was related to survival at day 28 and day 84. Follow-up was comprehensive, with reviews of medical records and contact with general practitioners if the outcome was uncertain. No patients were lost to follow-up. The model of end-stage liver disease (MELD) score was not calculated because at participating centres the prothrombin time was measured rather than the international normalised ratio (INR; which is not identical to the prothrombin time ratio). Therefore only the mDF and the GAHS could be calculated on the basis of the prolongation of prothrombin time and prothrombin time ratio, respectively.

The use of corticosteroids during the admission episode was noted. There was inevitably a degree of heterogeneity between the different hospitals as detailed in table 2; however, the corticosteroid-treated patients and untreated patients had similar admission characteristics (table 3 ). The corticosteroids were prescribed as prednisolone $40 \mathrm{mg}$ per day for four weeks, with the exception of King's College Hospital, London, where $30 \mathrm{mg}$ per day was administered. No patients received pentoxifylline or other specific therapies for alcoholic hepatitis.

Statistical analysis was performed using SigmaStat v.2.03, (SPSS Inc., Chicago, Illinois, USA). Comparisons of proportions were used to analyse differences between the groups for survival at day 28 and day 84 . A subgroup analysis of the data from King's College Hospital was performed as these patients were randomly assigned to receive corticosteroids as part of a trial. To complete the analysis, we tested whether the effects of treatment differed depending upon the stratification by GAHS. An initial multivariable logistic regression analysis of corticosteroid treatment (untreated $=0$ or treated $=1$ ) and GAHS $(<9=0, \geqslant 9=1)$ with 28 and 84 day survival as the outcome (death $=0$, survival $=1$ ) was performed. A further analysis was performed with these variables and an interaction term (GAHS ${ }^{*}$ corticosteroid treatment). The initial model assumed equal effect of treatment between the GAHS groups. The second analysis removed only that assumption. The models were then compared using a likelihood ratio test.

\section{RESULTS}

Of the 225 patients with an mDF greater than or equal to 32 and a calculable GAHS, 117 patients received corticosteroids (52\%). In total, $144(64 \%)$ patients had a GAHS greater than or equal to 9 , of whom 73 patients $(51 \%)$ received corticosteroids. Liver biopsy confirmation of the diagnosis of alcoholic hepatitis was available in 127 patients (56\%).

The overall 28-day survival was 79\% (92 patients) and $63 \%$ (68 patients) for those treated with corticosteroids and for those who did not receive corticosteroids, respectively $(\mathrm{p}=0.01$ corticosteroids compared with no treatment). The overall 84day survival was $62 \%$ (73 patients) and 50\% (54 patients) for those treated with corticosteroids and for those who did not receive corticosteroids, respectively $(p=0.09$ corticosteroids compared with no treatment).

Patients were subdivided into those with a GAHS less than or greater than or equal to 9. For patients with an admission GAHS less 9 there was no difference in survival between those patients treated with corticosteroids and those who did not receive treatment (35 patients, $80 \%$; and 31 patients, $84 \%$, respectively for day 28 survival (4\% difference, 95\% CI -12.7 , $20.7 \%$ ), and 30 patients, $68 \%$; and 27 patients, $73 \%$, respectively for day 84 survival ( $5 \%$ difference, $95 \%$ CI $-14.9,24.9 \%)$ ). For those patients with a GAHS greater than or equal to 9, however, there was a significant improvement in survival for patients

Table 3 Characteristics of corticosteroid-treated and untreated patients

\begin{tabular}{|c|c|c|c|c|}
\hline & \multicolumn{2}{|c|}{ Corticosteroid treated ( $n=117 ; 52 \%$ ) } & \multicolumn{2}{|c|}{ Untreated (n = 108; 48\%) } \\
\hline & GAHS $<9$ ( $n=44)$ & GAHS $\geqslant 9(n=73)$ & GAHS $<9$ (n = 37) & GAHS $\geqslant 9(n=71)$ \\
\hline Age & $46(12)$ & $46(9)$ & $44(10)$ & $46(10)$ \\
\hline Serum bilirubin ( $\mu \mathrm{mol} / \mathrm{I})$ & $308(171)$ & $475(161)$ & $262(141)$ & $452(181)$ \\
\hline Serum creatinine $(\mu \mathrm{mol} / \mathrm{l})$ & 149 (283) & $194(186)$ & $87(41)$ & $156(100)$ \\
\hline Blood urea $(\mathrm{mmol} / \mathrm{l})$ & $4.5(6)$ & $10.9(9.4)$ & $3.8(3.6)$ & $10.8(8.3)$ \\
\hline Prothrombin time ratio & $1.5(0.2)$ & $2.0(0.5)$ & $1.7(0.4)$ & $2.0(0.6)$ \\
\hline White cell count $\left(10^{9} / \mathrm{I}\right)$ & $10.1(4.1)$ & $17.5(8.2)$ & $11.2(5.2)$ & $17.4(9.5)$ \\
\hline GAHS & $8(6-8)$ & $10(9-12)$ & $8(6-8)$ & $10(9-12)$ \\
\hline $\mathrm{mDF}$ & $50.1(15)$ & $87.4(36.3)$ & $54.9(19.2)$ & $85.7(36.3)$ \\
\hline
\end{tabular}

GAHS, Glasgow alcoholic hepatitis score; mDF, modified Maddrey's discriminant factor.

Mean (standard deviation); (median and range for GAHS value).

There were no significant differences between corticosteroid-treated and untreated patients of each GAHS grouping. 

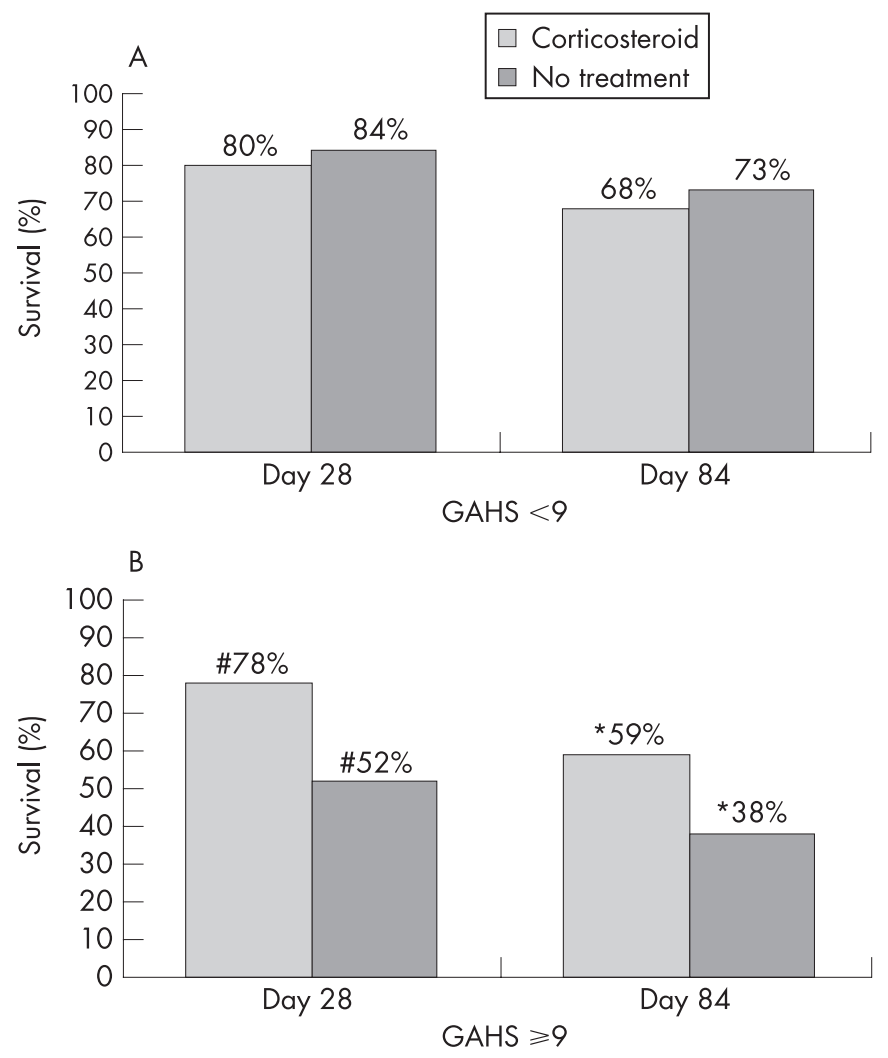

Figure 1 (A) Survival at day 28 and day 84 in patients with a modified Maddrey's discriminant factor (mDF) of 32 or greater and a Glasgow alcoholic hepatitis score (GAHS) less than 9: relative to the GAHS and corticosteroid treatment. (B) Survival at day 28 and day 84 in patients with an $\mathrm{mDF}$ of 32 or greater and a GAHS or 9 or greater: relative to the GAHS and corticosteroid treatment.

who received corticosteroids. Day 28 survival was $78 \%$ (57 patients) and 52\% (37 patients) for those treated with corticosteroids and for those who did not receive treatment, respectively ( $p=0.002$ corticosteroids compared with no treatment; $26 \%$ difference, 95\% CI 11, 41\%). Day 84 survival was 59\% (43 patients) and 38\% (27 patients) for those treated with corticosteroids and for those who did not receive treatment, respectively $(\mathrm{p}=0.02$ corticosteroids compared with no treatment; $21 \%$ difference, 95\% CI 5, 37\%; fig 1).

Initial multivariable logistic regression analysis without inclusion of the interaction term indicated that the higher GAHS group have a significantly reduced survival at 28 days (odds ratio (OR) $0.43 ; 95 \%$ CI $0.22,0.84 ; \mathrm{p}=0.01$ ). In addition, corticosteroid treatment was associated with improved survival at 28 days (OR $2.2 ; 95 \%$ CI $1.18,3.92 ; \mathrm{p}=0.01$ ). For the 84 -day outcome the GAHS retained significance (OR $0.4 ; 95 \%$ CI 0.22 , $0.72 ; \mathrm{p}=0.002)$ although this was lost for corticosteroid treatment (OR 1.64; 95\% CI 0.96, 2.83; p=0.07). With the inclusion of the interaction term, this term was significant at 28 days (OR 4.35; 95\% CI 1.13, 16.8; $\mathrm{p}=0.03$ ) but just loses significance at 84 days (OR 2.94; 95\% CI 0.91, 9.51; $\mathrm{p}=0.07$ ).
Comparison of likelihood values in table 4 shows that the full interaction model reflects the study data significantly more accurately than the non-interaction model at 28 days. At 28 days the odds ratio of corticosteroid treatment effect upon survival is 3.27 (95\% CI 1.59, 6.75) for patients with a GAHS greater than or equal to 9. For those with a GAHS less than 9, the odds ratio was 0.75 (95\% CI $0.24,2.35)$. This indicates that at 28 days the effect of treatment differs significantly between the two GAHS groups.

The subgroup analysis of patients from King's College Hospital, London, was performed as these patients were part of a randomised controlled trial, with corticosteroid treatment as one of the arms of the study. In patients with a GAHS greater than or equal to 9 , the 28 -day survival was $41 \%$ ( 11 patients) and $67 \%$ (18 patients) for those patients not treated with corticosteroids and those treated with corticosteroids, respectively (27 patients in each group; $\mathrm{p}=0.1$ ). The 28 -day survival for those patients with a GAHS less than 9 was $73 \%$ ( 8 out of 11 patients) and $76 \%$ ( 13 out of 17 patients) for those patients not treated with corticosteroids and those treated with corticosteroids, respectively.

\section{DISCUSSION}

Current recommendations from the American College of Gastroenterology are that corticosteroid treatment should be considered for patients with an $\mathrm{mDF}$ greater than or equal to $32 .{ }^{6}$ We have described the GAHS as an easily calculable method of assessing the severity of alcoholic hepatitis. The GAHS has a much higher specificity and overall accuracy than the mDF for outcome in alcoholic hepatitis. ${ }^{2}$ We have shown previously that a GAHS greater than 9 is able to identify patients with alcoholic hepatitis who have an especially poor prognosis. It is for this reason that this threshold was used to evaluate the response to corticosteroids in the current study. Recent studies have suggested using the MELD score to assess the severity of alcoholic hepatitis. In each of those studies, however, the MELD score on admission was not superior to the $\mathrm{mDF}^{9-11}$ on area under the curve analysis. In addition, none of those studies indicated whether the MELD score was a useful tool in identifying patients who may benefit from medical intervention. We were unable to calculate the MELD score in the current study as the INR was not specifically measured in this group of patients.

Although only applicable to patients with an mDF greater than or equal to 32 , our study indicates that in patients with a GAHS less than 9 there was no appreciable benefit with corticosteroid treatment. Patients with a GAHS greater than or equal to 9, however, have an extremely poor prognosis if they are not treated with corticosteroids, or if such treatment is contraindicated. The prognosis is significantly improved if they receive treatment or are eligible for such treatment. This was not a randomised trial and therefore the groups might not be comparable. In particular, patients with sepsis or active gastrointestinal bleeding who would not receive corticosteroid treatment might have a worse overall prognosis. Several observations, however, suggest that the comparisons are justified. First, the similar outcome of all patients with a

Table 4 Comparison of non-interaction and interaction models

\begin{tabular}{lllllll}
\hline & 28-Day outcome & & & & 84-Day outcome & \\
\cline { 2 - 3 } Model fit statistics & No interaction & Full interaction & & No interaction & Full interaction \\
\hline -2 log likelihood & $257.11^{*}$ & $252.46^{*}$ & & $294.70^{\#}$ & $291.41^{\#}$ \\
Degrees of freedom & 2 & 3 & & 2 & 3 & \\
\hline${ }^{*} \mathrm{p}=0.03 ;{ }^{*} \mathrm{p}=0.07$. & & & & \\
\hline
\end{tabular}


GAHS less than 9 suggests that no one treatment group had an excess mortality. Second, even though the subgroup analysis of the patients from the King's College controlled trial failed to reach significance (probably on account of the small numbers analysed), the trend was similar to that of the whole group. Although the time period of the King's College group differed from that of the other studies, it is improbable that this would influence the outcome of these patients. Third, the 28-day survival of the untreated patients and the corticosteroid-treated patients in this study was similar to that seen on the cumulative analysis of the three most recent randomised controlled trials of corticosteroid treatment in alcoholic hepatitis. ${ }^{5}$ Therefore, we believe that these comparisons are justified, although a further prospective study should be performed to confirm this.

The current study indicates that patients with a low GAHS do not appear to benefit from corticosteroids. Previous trials and meta-analyses of corticosteroid therapy in alcoholic hepatitis have given apparently contradictory results. ${ }^{3}$ One reason for this may be that the common entry criteria of an mDF greater than or equal to 32 is not specific enough for mortality to allow a clear beneficial effect of treatment to be seen. The GAHS is more specific for mortality than the mDF. Using a GAHS greater than or equal to 9 as the criteria for entry, future trials might be better placed to identify any benefit from pharmacological therapy.

In conclusion, the GAHS is a validated scoring system for the assessment of alcoholic hepatitis that appears to be able to identify patients with an $\mathrm{mDF}$ greater than or equal to 32 who may benefit from corticosteroid treatment.

\section{ACKNOWLEDGEMENTS}

The authors would like to thank Alan H Forrest, PhD, for statistical advice.

\section{Authors' affiliations}

E H Forrest, A J Morris, Glasgow Royal Infirmary, Glasgow, UK

S Stewart, C P Day, University of Newcastle, Newcastle-upon-Tyne, UK

M Phillips, J O'Grady, King's College Hospital, London, UK

Y H Oo, G Haydon, University Hospital, Birmingham, UK

N C Fisher, Dudley Hospitals, Dudley, West Midlands, UK

Conflict of interest: None declared.

\section{REFERENCES}

1 Carithers JRL, Herlong HF, Diehl AM, et al. Methylprednisolone therapy in patients with severe alcoholic hepatitis: a randomized multicenter trial. Ann Intern Med 1989;1 10:85-690.

2 Forrest EH, Evans CDJ, Stewart S, et al. Analysis of factors related to mortality in alcoholic hepatitis and the derivation and validation of the Glasgow alcoholic hepatitis score. Gut 2005;54:1174-9.

3 Morgan M. The treatment of alcoholic hepatitis. Alc Alcoholism 1996;31:117-34.

4 Christensen E. Alcoholic hepatitis - glucocorticoids or not? J Hepatol 2002;36:547-8.

5 Mathurin P, Mendenhall CL, Carithers J, et al. Corticosteroids improve short-term survival in patients with severe alcoholic hepatitis $(\mathrm{AH})$ : individual data analysis of the last three randomized placebo controlled double blind trials of corticosteroids in severe AH. J Hepatol 2002;36:480-7.

6 McCullough AJ, O'Connor JFB. Alcoholic liver disease: proposed recommendations for the American College of Gastroenterology. Am J Gastroenterol 1998;93:2022-36.

7 Stewart SF, Prince M, Bassendine MF, et al. A randomized trial of antioxidant therapy alone or with corticosteroids in acute alcoholic hepatitis. $J$ Hepatol 2007; 47:277-83.

8 Phillips M, Curtis H, Portmann B, et al. Antioxidants versus corticosteroids in the treatment of severe alcoholic hepatitis - a randomised clinical trial. J Hepatol 2006:44:784-90.

9 Sheth M, Riggs M, Patel T. Utility of the Mayo end-stage liver disease (MELD) score in assessing prognosis of patients with alcoholic hepatitis. BMC Gastroenterol 2002;2:2.

10 Dunn W, Jamil LH, Brown LS, et al. MELD accurately predicts mortality in patients with alcoholic hepatitis. Hepatology 2005;41:353-8.

11 Srikureja W, Kyulo NL, Runyon BA, et al. MELD is a better prognostic model than the Child-Turcotte-Pugh score or Discriminant Function in patients with alcoholic hepatitis. J Hepatol 2005;42:700-6. 